\title{
Poynting Flux Dominated Black Hole-Accretion Disk System as GRB Power House
}

\author{
Hyun Kyu Lee and Hui-Kyung Kim \\ Department of Physics, Hanyang University, Seoul 133-791, Korea
}

\begin{abstract}
We investigate a black hole-accretion disk system which is dominated by Poynting flux to see whether it can be a possible power house for GRBs. For a set of GRBs for which the isotropic energy and $T_{d}$ are known, the effect of the disk mass and the magnetic field are discussed quantitatively using a simplified model. It is demonstrated explicitly that there is a lower limit on the angular momentum parameter for a given GRB energy. It is found that the most energetic GRBs can only accommodate relatively rapid-rotating black holes at the center.
\end{abstract}

\section{Introduction}

It is generally accepted that the central region powering gamma ray burst is rather compact in size, less than $10^{8} \mathrm{~cm}$, from the study of energies and temporal structures of GRB and afterglows followed afterwards (Piran 1999). Merging binary compact objects, hypernovae and rotating black holes have been considered to be among the viable candidates for a GRB central engine. The common feature of these models is the formation or the existence of a black hole at the central region. However, the gamma ray bursts or the afterglows followed do not provide any direct observational informations on the central region and we do not know the exact nature of the black hole at the center. Hence although it is very important and interesting to investigate the central object itself, we should rely on the indirect method of using a particular model, in which the physical properties of black holes can be inferred from the observational data.

In this work we choose a model in which the rotational energy of the black hole is responsible for powering GRB(Lee, Brown, \& Wijers 2000). The mechanism of tapping rotational energy from the black hole has been known as Blandford-Znajek mechanism(Blandford \& Znajek 1977; Thorne, Price, \& Mcdonald 1986), in which the rotational energy is extracted out to the loading region via the magnetic flux which threads on the horizon. It is easy to demonstrate that the strong magnetic field of $\sim 10^{15}$ gauss is consistent with the essential features of GRBs. It is well known that the black hole itself cannot keep the magnetic field on the horizon. It disappears very rapidly if not the environment keeps them from disappearing. Recently, it is shown that the magnetic flux on the strongly magnetized object can be maintained during the collapsing process together with the electric charge onto the black hole(Lee, Lee, \& van Putten 2001). When the gravitationally unstable object collapses into black hole the most natural environment is the accretion disk/torus which emerges together 
with the central black hole and it can provide the magnetoshpere which keeps the magnetic flux from disappearing. Because of the accretion the energy and the angular momentum are carried into the black hole while the Poynting flux carries away energy and angular momentum out of the black hole(Lee, Brown, \& Wijers 2000), the evolution of a black hole depends not only on the Poynting flux but also on the accretion.

In this work we make an attempt to infer the evolution of the central black hole during the gamma rays are bursting using a simple model of a black holeaccretion disk system suggested by Lee and $\operatorname{Kim}(2000)$ in which the accretion is dominated by the Poynting flux(Blandford 1976; Lee 2001). In section 2, the simple minded model is sketched with emphasis on the parameters which govern the evolution of the black hole. We take the initial values of the magnetic field on the horizon and the disk mass as parameters while the black hole mass is initially taken to be a typical mass, $7 M_{\odot}$, suggested from the black hole binary systems. The evolution of the angular momentum parameter $\tilde{a}$ is discussed also in detail. With finite mass of accretion disk, $M_{D} \leq 7 M_{\odot}$, the initially rapidrotating black hole is found to remain rotating even at the end of GRB. But the initially slow-rotating black hole is found to eventually stop rotating with GRB. In section 3, the total energy out of the rotating black hole is calculated and compared to GRBs for which the isotropic energies are known(Bloom, Frail, \& Sari 2001) . The lower limits of the angular momentum parameter of the black holes for the corresponding GRBs are calculated. For the most energetic GRBs, $E_{i s o}>.1 M_{\odot}$, the lower limit is found to be rather high, $\tilde{a}(0)>0.3$. The evolution of the black hole is also discussed for GRBs with known $T_{d}$. Identifying $E_{i s o}$ as $90 \%$ of energy extraction and $T_{d}$ as the time taken to extract out $E_{i s o}$, the corresponding sets of $B_{H}(0)$ and $M_{D}(0)$ are determined to discuss the evolution of the corresponding black holes. The discussions are given in section 4 .

\section{A simple model of black hole - accretion disk system}

In this work, the the energy of GRB is supposed to be powered by Poynting flux out of the rotating black hole surrounded by the magnetized accretion disk (Lee, Wijers, \& Brown 2000). To see the effects of the magnetic field on the evolution of the black-hole-accretion disk, it is assumed that the accretion flow is driven mainly by the strong magnetic field which carries angular momentum by Poynting flux (Blandford 1976; Lee \& Kim 2000, 2002). Then we can have an analytic formulation of the black hole evolution. The essential feature of the evolution model discussed is briefly reviewed in the following.

The evolution rates of the black hole mass $(\dot{M})$ and the angular momentum $(\dot{J})$ are determined both by the energy and the angular momentum accreted, which increase the mass and the angular momentum, and also by the BlandfordZnajek power in the opposite direction. From the energy and angular momentum conservation, we get the rates of change for the mass and the angular momentum given by

$$
\begin{aligned}
\dot{M} & =-P_{B Z}+\dot{M}_{+} \tilde{E} \\
\dot{J} & =-\frac{P_{B Z}}{\Omega_{F}}+\dot{M}_{+} \tilde{l}
\end{aligned}
$$


Table 1. The isotropic energies of GRBs and the lower limits of angular momentum parameter, $\tilde{a}_{l}$.

\begin{tabular}{|c|c|c|c|c|}
\hline GRB & $\mathrm{z}$ & $d_{L}[\mathrm{~cm}]$ & $E_{\text {iso }}[\mathrm{erg}]$ & $\tilde{a}_{l}$ \\
\hline 970228 & 0.695 & $1.403 \mathrm{e}+28$ & $1.42 \mathrm{e}+52$ & 0.14 \\
\hline 970508 & 0.835 & $1.757 \mathrm{e}+28$ & $5.46 \mathrm{e}+51$ & 0.09 \\
\hline 970828 & 0.958 & $2.082 \mathrm{e}+28$ & $2.20 \mathrm{e}+53$ & 0.31 \\
\hline 971214 & 3.418 & $9.877 \mathrm{e}+28$ & $2.11 \mathrm{e}+53$ & 0.31 \\
\hline 980613 & 1.0969 & $2.459 \mathrm{e}+28$ & $5.67 \mathrm{e}+51$ & 0.09 \\
\hline 980703 & 0.9662 & $2.103 \mathrm{e}+28$ & $6.01 \mathrm{e}+52$ & 0.21 \\
\hline 990123 & 1.6004 & $3.925 \mathrm{e}+28$ & $1.44 \mathrm{e}+54$ & 0.45 \\
\hline 990506 & 1.30 & $3.037 \mathrm{e}+28$ & $8.54 \mathrm{e}+53$ & 0.42 \\
\hline 990510 & 1.619 & $3.982 \mathrm{e}+28$ & $1.76 \mathrm{e}+53$ & 0.30 \\
\hline 990705 & 0.84 & $1.770 \mathrm{e}+28$ & $2.70 \mathrm{e}+53$ & 0.33 \\
\hline 990712 & 0.433 & $7.927 \mathrm{e}+27$ & $5.27 \mathrm{e}+51$ & 0.09 \\
\hline 991208 & 0.707 & $1.429 \mathrm{e}+28$ & $1.47 \mathrm{e}+53$ & 0.28 \\
\hline 991216 & 1.02 & $2.250 \mathrm{e}+28$ & $5.35 \mathrm{e}+53$ & 0.39 \\
\hline 000131 & 4.500 & $1.369 \mathrm{e}+29$ & $1.16 \mathrm{e}+54$ & 0.44 \\
\hline $000301 \mathrm{C}$ & 2.034 & $5.269 \mathrm{e}+28$ & $4.64 \mathrm{e}+52$ & 0.19 \\
\hline 000418 & 1.118 & $2.523 \mathrm{e}+28$ & $8.29 \mathrm{e}+52$ & 0.24 \\
\hline 000926 & 2.037 & $5.280 \mathrm{e}+28$ & $2.97 \mathrm{e}+53$ & 0.34 \\
\hline
\end{tabular}

where $\dot{M}_{+}$is the mass accretion rate and the specific energy and angular momentum of the accreting matter are denoted as $\tilde{E}$ and $\tilde{l}$ respectively. It is assumed that the inner edge of the accretion disk is the last stable orbit(Shapiro \& Tuekolsky 1983). Since the presence of an accretion disk with an appreciable pressure and/or magnetic field is essential for the magnetic field on the horizon, the evolution of the accretion disk is also responsible for the evolution of the magnetic field on the disk and $B_{H}$.

For the numerical calculations in this work, we assume that the effects of mass loss from the disk can be incorporated into the the time dependence of the magnetic field in the following form:

$$
B_{H}^{2}=B_{H}^{2}(0) D(t), \quad D(t)=1-\left(\int_{0}^{t} \dot{M}_{+}\right) / M_{D}(0)
$$

We take $D(t)$ to be vanishing as the total accreting mass becomes the initial disk mass, which is supposed be typically of solar mass for an accretion disk emerging out of binary - merging processes.

The rotation of a black hole can be described either by angular momentum $J$, or specific angular momentum $a(=J / M)$ or angular momentum parameter $\tilde{a}(=a / M)$. In this work we will take the angular momentum parameter $\tilde{a}$ which is a dimensionless quantity to represent the rotation of the black hole. The evolution of the angular momentum parameter $\tilde{a}$ can be obtained from the 
angular momentum evolution as

$$
\frac{\dot{J}}{J}=\frac{\dot{a}}{a}+\frac{\dot{M}}{M}
$$

Then, we get

$$
\dot{\tilde{a}}=A \frac{\dot{M}_{+}}{M}
$$

where the sign factor $A$ defined by

$$
A=\left(\mathcal{Z}_{l}-\tilde{P}_{l}\right)-2 \tilde{a}\left(\tilde{E}-\tilde{P}_{E}\right)
$$

determines whether $\tilde{a}$ is decreasing or increasing. Numerical calculation shows that the sign factor is negative for the entire range of $\tilde{a}$. It means that the rate of change of the angular momentum parameter $\dot{\tilde{a}}$, is always negative in this model described in the previous section. It should be noted that this sign factor is independent of the parameters of the model. It is the intrinsic feature of the model. The sign factor has a maximum at $\tilde{a} \rightarrow \tilde{a}_{m}=.57$ but still less than zero(Lee \& Kim 2000). It is analogous to the energy barrier to be overcome in particle dynamics. If the evolution starts with $\tilde{a}>.57$ one should wait long time until the black hole stops rotating. It is because when it is crossing the maximum value, $\tilde{a}_{m}$, the process becomes very slow. With the finite mass of the accretion disk, the evolution ends when the disk disappears completely into the black hole. In this case however the evolution may end up with the rotating black hole as one can see in Fig. 1. On the other hand, if the evolution starts with $\tilde{a}<.57$ the slow-down process accelerates in the beginning and the black hole eventually stops rotating in a much shorter time. However it depends also on the evolution of the disk. Since the life time of the disk depends essentially on the initial mass, we can define, for a given mass of accretion disk, $\tilde{a}_{c}$ : a black hole start with $\tilde{a}<\tilde{a}_{c}$ ends up with non-rotating black hole.

\section{Gamma ray burst and evolution of black hole}

The energy of GRB is supposed to be powered by the Poynting flux out of the rotating black hole at the center of a black hole - accretion disk system. Essentially the energy is a part of the rotational energy of the black hole. The rotational energy of the disk is accreted into the black hole and then it is processed to be a part of the black hole's rotational energy. Therefore the source of the energy delivered to GRB is not only the black hole's rotational energy but also the rotational energy of the disk.

The duration of the GRB is identified to be the duration of Poynting flux from the system. The Blandford-Znajek process becomes ineffective either when there is no magnetic field on the black hole or it does not rotate. If the system starts with the larger disk mass, the life time is longer for a given initial magnetic field. Therefore the energy carried out until the Blandford-Znajek process stops is expected to be larger for the large disk mass system. Numerical calculations in Fig. 2 shows that the energy is increasing with the initial disk mass, $M_{D}(0)$. 


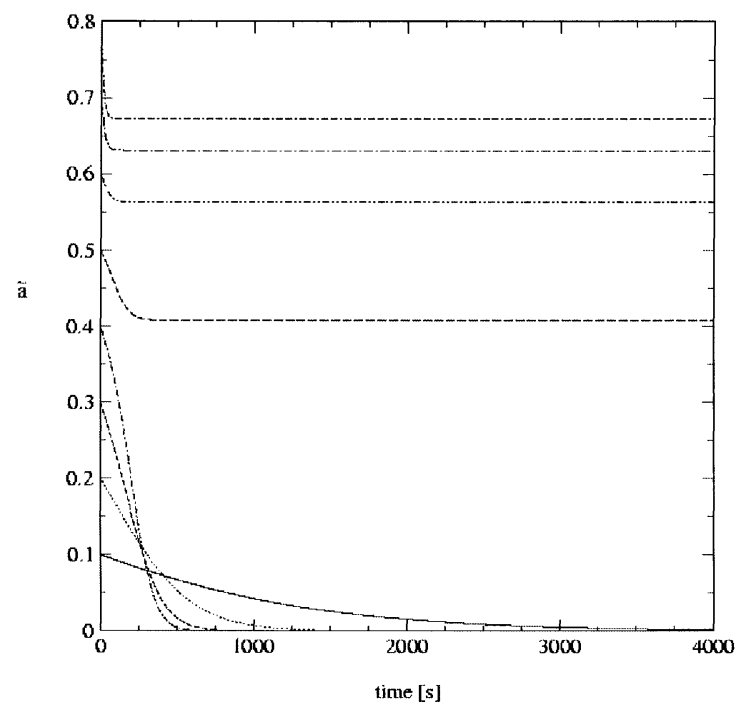

Figure 1. The evolution of the angular momentum parameter in time with $M(0)=7 M_{\odot}$ and $M_{D}(0)=3 M_{\odot}$.

However for $\tilde{a}<\tilde{a}_{c}$, the energy reaches maximum at a certain value of disk mass, $M_{D}^{c}$, and shows little change for the larger disk mass. It is because the rotation of the black hole slows sufficiently down for the Blandford-Znajek process to be ineffective as discussed in the previous section. When the system starts with $M_{D}(0)>M_{D}^{c}$, the black hole is slow down substantially before the life time of the accretion disk. And the GRB duration is determined by the time when black hole stops rotating not by the life time of the disk. The radial current in the disk vanishes and there is no magnetic breaking hence no accretion onto the black hole. For $\tilde{a}>\tilde{a}_{c}$, the disk disappears into the black hole while the remaining black hole is still rotating. But there is no Poynting flux because the magnetic field supported by the disk disappears altogether. Then the duration of GRB is determined by the disk life time. Compared to the isotropic energies of GRB, one can estimate the lower limits of the initial angular momentum parameters of the black holes at the center of the GRB in this model, Table 1. One can see that most of energetic GRBs require rapidly rotating black holes at the center, $\tilde{a}(0) \geq 0.3$.

The effect of the magnetic field on the total energy out of the system is found to be not so significant(Lee \& Kim 2002). But the strength of the magnetic field is directly involved in determining the rate of energy carried out along the magnetic flux. The stronger magnetic field extracts energy more rapidly than the weaker magnetic field. Hence the detailed variation of the GRB duration is expected to be related to the magnetic field structure of the system. In this work it is parameterized by the initial value on the horizon, $B_{H}(0)$.

The determination of the duration of GRB from the observational data is relatively complicated than the energy. In this work, we take $T_{d}$ as duration time 
for $90 \%$ of the total Poynting flux energy to be carried out. The general feature of the result does not change significantly from the previous study with $T_{90}$ (Lee \& Kim 2002). Among the GRBs with inferred isotropic energies in Table 1, we choose 10 of them for which $T_{d}$ s are well defined, Table 2.

The evolution of a black hole is determined by the mass and angular momentum. For the system with $\tilde{a}(0)=.8$, it is found that the final mass of the black holes are found to be increasing: $\Delta M_{B H}=.01 M_{\odot}$ up to $2.8 M_{\odot}$. Since $\tilde{a}(0)=.8>\tilde{a}_{m}$, the final black holes are expected to be rotating even after GRB with smaller angular momentum parameters than the initial values. For $\tilde{a}(0)=.3$, which is smaller than the lower limits of GRB 970828, 971214, 990123, 991216,000131 which require more rapidly rotating black holes at the center, the less energetic GRBs can accommodate the slowly rotating black holes. In this case, however, the appropriate disk mass is found to be smaller than $M_{D}^{c}$ and the black hole remains rotating after GRB. It is because $\tilde{a}(0)$ is greater than $\tilde{a}_{c}$ for the corresponding disk mass.

Table 2. The initial mass and magnetic field of black hole for GRBs for which $T_{d}(\mathrm{~s})$ are well defined. $M_{B H}^{f}$ and $\tilde{a}^{f}$ are the final black hole mass and the angular momentum parameter respectively. Magnetic field and mass are in the unit of $10^{15}$ gauss and $M_{\odot}$ respectively. (1)970228, (2)970508, (3)970828, (4)971214, (5)980613, (6)980703, (7)990123, (8)990510, (9)991216, (10)000926

\begin{tabular}{|c|c|c|c|c|c|c|c|c|c|c|c|}
\hline \multicolumn{2}{|c|}{ GRB } & 1 & 2 & 3 & 4 & 5 & 6 & 7 & 8 & 9 & 10 \\
\hline$\tilde{a}(0)$ & $T_{d}$ & 1.75 & 6.3 & 25.84 & 1.78 & 2.35 & 11.27 & 16.17 & 3 & 11.44 & 9.98 \\
\hline \multirow{3}{*}{1.} & $B_{H}(0)$ & 1.46 & 0.472 & 1.533 & 5.75 & 0.795 & 1.184 & 5.144 & 3.318 & 3.378 & 2.927 \\
\cline { 2 - 12 } & $M_{D(0)}(0)$ & 0.012 & 0.004 & 0.33 & 0.320 & 0.004 & 0.066 & 3.280 & 0.159 & 0.816 & 0.497 \\
\cline { 2 - 12 } & $M_{B H}^{f}$ & 6.999 & 6.999 & 7.100 & 7.096 & 6.999 & 7.008 & 8.717 & 7.035 & 7.326 & 7.173 \\
\cline { 2 - 12 } & $\tilde{a}^{f}$ & 0.998 & 0.999 & 0.958 & 0.959 & 0.999 & 0.989 & 0.804 & 0.977 & 0.917 & 0.943 \\
\hline \multirow{3}{*}{0.8} & $B_{H}(0)$ & 2.235 & 0.725 & 2.208 & 8.378 & 1.228 & 1.802 & 6.152 & 4.949 & 4.64 & 4.162 \\
\cline { 2 - 11 } & $M_{D}(0)$ & 0.045 & 0.017 & 0.692 & 0.672 & 0.018 & 0.189 & 4.359 & 0.386 & 1.409 & 0.952 \\
\cline { 2 - 11 } & $M_{R H}^{f}$ & 7.027 & 7.010 & 7.424 & 7.416 & 7.011 & 7.116 & 9.753 & 7.238 & 7.874 & 7.589 \\
\cline { 2 - 12 } & $\tilde{a}^{f}$ & 0.798 & 0.799 & 0.777 & 0.778 & 0.799 & 0.793 & 0.706 & 0.787 & 0.758 & 0.770 \\
\hline \multirow{3}{*}{0.3} & $B_{H}(0)$ & 6.525 & 2.106 & & & 3.56 & 5.496 & & 16.56 & & \\
\cline { 2 - 11 } & $M_{D}(0)$ & 0.067 & 0.026 & & & 0.272 & 0.286 & & 0.588 & & \\
\cline { 2 - 12 } & $M_{B H}^{f}$ & 7.049 & 7.018 & & & 7.020 & 7.206 & & 7.425 & & \\
\cline { 2 - 11 } & $\tilde{a}^{f}$ & 0.293 & 0.298 & & & 0.297 & 0.269 & & 0.223 & & \\
\hline
\end{tabular}

\section{Discussion}

Using a simple model for a central engine of GRB, which consists of a black hole and an accretion disk dominated by Poynting flux (Lee \& Kim 2000, Lee \& Kim 2002), the characteristics of the system have been studied and also the evolution of the central black hole is discussed. The accretion is assumed to be dominated by the Poynting flux out of the disk and the GRB is supposed to be powered by the Poynting flux which extracts out a part of the rotational energy of a black hole. It is found that the evolution of the rotation parameterized by $\tilde{a}(t)$ shows different patterns depending on the initial value, $\tilde{a}(0)$. For a given disk mass, the black hole with $\tilde{a}(0)<.57$ much more rapidly approaches to non-rotating black hole than with $\tilde{a}(0)>.57$. It is also shown that there is a maximum energy for GRB for a given $\tilde{a}(0)$. Hence one can infer the lower limit of the angular 


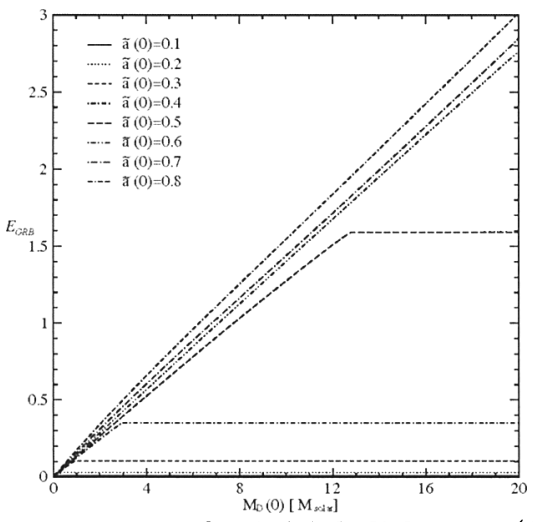

Figure 2. The energy vs. the initial disk $\operatorname{mass}\left(M_{D}(0)\right)$ for various initial angular momentum parameters $(\tilde{a}(0))$.

momentum parameter for the central black hole. Most of energetic GRBs are found to be able to accommodate only rapidly rotating black holes, $\tilde{a}(0)>0.3$.

The effect of the magnetic field on the total energy for GRB is found to be not significant compared to the disk mass. However since the stronger magnetic field extract energy more rapidly than the weaker magnetic field, the detailed variation of the GRB duration is found to be due to the magnetic field structure of the system. The role of the disk mass in this model is significant both in determining the energy and the duration of GRB. Within the range of the parameters used to fit a set of GRBs for which isotropic energy and $T_{d}$ are well determined, the final black holes are found to become more massive than the initial values but with smaller angular momentum parameters. This observation is consistent with the general feature expected for a system of a black hole and a accretion disk with finite size and life time. As it is mentioned the analysis has been limited to GRBs with $T_{90}$ and/or $T_{d}$. But provided with a systematic way of determining GRB duration time from the observational data, statistically the more meaningful conclusion can be made on the evolution of the black hole at the center of GRB.

In this work, several simplification which are subject to be verified have been made. For example, we use the relation of the field components suggested by Blandford(1976) which requires a justification if it can be used in the relativistic formulation especially for a rapidly rotating black hole. Also the identification of the angular velocity of the field line as the Keplerian angular velocity in this model also needs a valid justification(Lee 2001).

Recent analysis of GRB shows the evidences of beaming in gamma ray bursts. Then the whole energy budget should be reduced by more than a factor of hundred up to thousand compared to the inferred isotropic energy. The simplest way to accommodate the effect of beaming into this model is to assume that the total Poynting power out of the system is beamed. Then it is easily expected that the disk mass should be reduced significantly to explain the reduced energy budget. As an example, for GRB990123 with beaming angle 0.050, the initial disk mass is found to be $0.01 M_{\odot}$ and the magnetic field $0.36 \times 10^{15}$ gauss which 
are much smaller than those for isotropic energy. However, in this case, it is not clear whether the whole Poynting power out of black hole should go into GRB. Some fraction may be transferred into the disk via magnetic coupling. The analysis on the beamed GRB remains as a future work.

\section{References}

Blandford, R. 1976, MNRAS, 176, 465

Blandford, R. D. \& Znajek, R. L. 1977, MNRAS, 179, 433

Bloom, J. S., Frail, D. A., \& Sari, R. 2001, AJ, 121, 2789

Lee, H. K. 2001, Phys.Rev.D, 64, 043006

Lee, H. K., Brown, G. E. \& Wijers, R. A. M. J., 2000, ApJ, 536, 416

Lee, H. K. \& Kim, H. K. 2000, J. Korean Phys. Soc., 36, 188

Lee, H. K. \& Kim, H. K. 2002, J. Korean Phys. Soc., 40, 524

Lee, H. K., Lee, C. H., \& van Putten, M. H. P. M. 2001, MNRAS, 324, 781

Lee, H. K., Wijers, R. A. M. J., \& Brown, G. E. 2000, Phys. Rep., 325, 83

Piran, T. 1999, Phys. Rep., 314, 576

Shapiro, S.L. and Teukolsky, S.A. 1983, Black Holes, White Dwarfs, and Neutron Stars (Wiley-Interscience Pub., New York)

Thorne, K. S., Price, R. H., \& MacDonald, D. A. 1986, Black Holes: The Membrane Paradigm (Yale University Press, New Haven and London) 